\title{
Impact of Desloratadine and Loratadine on the Crosstalk between Human Keratinocytes and Leukocytes: Implications for Anti-Inflammatory Activity of Antihistamines
}

\author{
C. Traidl-Hoffmann I. Münster J.Ring H. Behrendt \\ Division of Environmental Dermatology and Allergy GSF/TUM, ZAUM - Center for Allergy and Environment, \\ Munich, Germany
}

\section{Key Words}

Antihistamine $\cdot$ Chemotaxis $\cdot$ Desloratadine $\cdot$

Keratinocytes $\cdot$ Loratadine

\section{Abstract}

Background: Desloratadine is an $\mathrm{H}_{1}$-histamine antagonist which possesses additional anti-inflammatory properties through inhibition of leukocyte activation and reduction of ICAM-1 expression on mucosal epithelial cells. So far no studies have addressed the potential anti-inflammatory activities of desloratadine and loratadine on skin keratinocytes. Objective: In this study the capacity of desloratadine and loratadine to counteract human keratinocyte activation by interferon- $\gamma$ (IFN- $\gamma$ ) was analyzed. In particular, the chemokine release of kerationcytes and the crosstalk between keratinocytes and lymphocytes were examined. Method: Keratinocyte cultures established from normal skin of healthy donors were activated by IFN- $\gamma$ in the absence or presence of desloratadine and loratadine, and tested for the release of CCL5/ RANTES, CXCL8/IL-8, CCL17/TARC and CXCL10/IP-10. Furthermore the supernatants of differentially stimulated keratinocytes were used for migration studies of human neutrophils, eosinophils and polarized Th1/Th2 clones. Results: Desloratadine and loratadine inhibited the constitutive and IFN- $\gamma$-induced release of CCL5, CXCL8 and CXCL10 from keratinocytes, while the low release of CCL17 remained un-
\end{abstract}

changed. Furthermore the crosstalk between lymphocytes and keratinocytes was blocked as shown by a reduced capacity of desloratadine/loratadine-stimulated keratinocytes to attract human neutrophils, eosinophils and T cells. Conclusions: The results indicate that desloratadine has the capacity to block the IFN- $\boldsymbol{\gamma}$-induced activation of keratinocytes, and that it can thus exert important regulatory effects on cell-mediated immune responses in the skin. The rather high doses required for these effects argue for a topical application when trying to use desloratadine in epidermal inflammatory conditions.

\section{Introduction}

Histamine $\mathrm{H}_{1}$-receptor antagonists have a long history of clinical efficacy in a variety of allergic inflammatory disorders. Desloratadine and loratadine are new-generation antihistamines that lack central nervous system and cardiovascular side effects $[1,2]$. In the recent past the basic understanding of the therapeutic effects of these modern antihistamines has been extended by the discovery of various potential anti-inflammatory actions other than the blockade of histamine receptors $[3,4]$. In these studies no correlation was seen between the potency of $\mathrm{H}_{1}$-receptor-blocking activity and the inhibition of hista- 
mine release, suggesting that a mechanism other than receptor antagonism was at work.

The skin is a frequent site of chronic inflammatory disorders such as allergic contact dermatitis, eczema and psoriasis. In these conditions the skin is infiltrated by migrating $\mathrm{T}$ lymphocytes and granulocytes which can exert direct cytotoxic effects and produce cytokines with proinflammatory functions. In particular interferon- $\gamma$ (IFN$\gamma)$ is an essential cytokine to amplify inflammatory reactions $[5,6]$, since it stimulates the synthesis of chemokines in keratinocytes that attract inflammatory cells and induces the expression of molecules important for the retention and activation of $\mathrm{T}$ cells $[7,8]$. The inhibition of the expression, production and secretion of these mediators is likely to provide a major tool for the treatment of inflammatory skin reactions [9]. In this study we sought to determine whether desloratadine and loratadine are able to counteract the effects of IFN- $\gamma$ on cultured human keratinocytes in terms of chemokine release and granulocyte or lymphocyte chemoattraction.

\section{Materials and Methods}

\section{Reagents and Antibodies}

Recombinant human IFN- $\boldsymbol{\gamma}$ was obtained from Genzyme (Cambridge, Mass., USA). Desloratadine and loratadine were provided by Essex Pharma (Schering-Plough, USA) and dissolved in ethanol. Anti-CD16, anti-CD4 and anti-CD45RA mAb microbeads were from Miltenyi Biotec (Bergisch-Gladbach, Germany).

\section{Culture and Stimulation of Keratinocytes}

Skin was obtained from foreskins of nonatopic donors $(n=5)$ and cultured as previously described [10]. The keratinocytes were prestimulated (keratinocyte growth medium, Clonetics, without hydrocortisone) with desloratadine or loratadine for $4 \mathrm{~h}$ and subsequently stimulated with IFN- $\gamma 300 \mathrm{U} \cdot \mathrm{ml}^{-1}$. The supernatants were taken after $24 \mathrm{~h}$ for ELISA analysis. For migration studies stimulated keratinocytes were washed after $24 \mathrm{~h}$ and incubated for another $24 \mathrm{~h}$ in keratinocyte growth medium w/o hydrocortisone. Thus the desloratadine/loratadine-free supernatants were harvested after $48 \mathrm{~h}$.

\section{ELISA Assays}

The chemokines were measured on cell-free supernatants collected after $24 \mathrm{~h}$ of treatment of subconfluent keratinocyte cultures in $1.9-\mathrm{cm}^{2}$ wells. The keratinocyte cultures were carried out in triplicate for each condition. The following sandwich ELISA kits from Becton Dickinson (Heidelberg, Germany) were used according to the instructions of the provider: CXCL8 (IL-8), CCL5 (RANTES), CXCL10 (IP10) and CCL17 (TARC).

Preparation of Granulocytes and Migration Assay

The granulocytes were isolated from human peripheral blood of nonatopic donors. The eosinophils were purified by magnetic- activated cell separation (MACS) as described previously [11]. The chemotactic activity of the keratinocyte supernatants was evaluated measuring the neutrophil or eosinophil migration through a $5-\mu \mathrm{m}$ pore polycarbonate filter in 24-well transwell chambers (Corning Costar, Cambridge, Mass., USA) as previously described [12]. The results are shown as total migrated cells.

\section{Generation of Polarized Th1 and Th2 Cell Clones}

Human CD4+, CD45RA+ T cells were purified from nonadherent peripheral blood mononuclear cells from healthy nonatopic donors using MACS column separators with anti-CD4 and anti-CD45RA microbeads (Miltenyi Biotec). Differently stimulated monocyte-derived dentric cells (DC; $24 \mathrm{~h}$ ) were washed and cocultured with MACS-purified allogeneic naive CD4+ and CD45RA+ T cells $\left(1 \times 10^{5}\right.$ cells/well $)$ in complete RPMI with $5 \%$ human serum. Lipopolysaccharide-activated DC (24 h) were used to generate Th1-polarized T cell lines. Th2-polarized T cells were generated by using DC that were activated $(24 \mathrm{~h})$ with lipopolysaccharide in the presence of $\mathrm{PGE}_{2}\left(10^{-6} \mathrm{M}\right.$; Alexis, San Diego, Calif., USA). In addition neutralizing anti-IL-12 mAb $(10 \mu \mathrm{g} / \mathrm{ml}$, $\mathrm{BD}$, Heidelberg, Germany) was added at the beginning of the $\mathrm{T}$ cell/DC coculture in order to generate a maximal Th2 polarization. The $\mathrm{T}$ cell lines were cloned after 10 days by limited dilution. In the migration assay the used Th1 clones were strongly positive for CXCR3 and CCR5 and negative for CCR4 and CRTh2, while the Th2 clones were negative for CCR5 and CXCR3 and positive for CCR4 and CRTh2 (data not shown; all antibodies from $\mathrm{BD}$ ).

\section{Propidium Staining}

The toxicity of the substances was evaluated by propidium iodide (PI) staining. Cells were collected, washed twice with cold PBS containing PI $\left(20 \mu \mathrm{g} \cdot \mathrm{ml}^{-1}\right)$. The toxicity was analyzed as PI-positive cells by flow cytometry (FACSCalibur, BD, USA).

\section{Statistical Analysis}

The Student t test was used to compare differently stimulated keratinocytes. A p value $<0.05$ was considered to be significant.

\section{Results}

Desloratadine and Loratadine Inhibit the Release of Constitutive or IFN- $\gamma$-Induced CXCL8, CXCL10 and CCL5 from Primary Human Keratinocytes

Supernatants of differently stimulated keratinocytes were analyzed for CCL5, CXCL10 and CXCL8. As shown in figure 1 desloratadine and loratadine dose-dependently inhibited the constitutive and induced releases of all 3 chemokines. The $\mathrm{IC}_{50}$ for the IFN- $\gamma$-induced CCL 5 was $3 \mu \mathrm{M}$ for loratadine and $10 \mu \mathrm{M}$ for desloratadine, whereas the $\mathrm{IC}_{50}$ s for the the IFN- $\gamma$-induced chemokines CXCL10 and CXCL8 did not differ between desloratadine and loratadine. Further differences were seen in the effects of the antihistamines on the constitutive expression of the chemokines, with CXCL10 being the most resistant to 


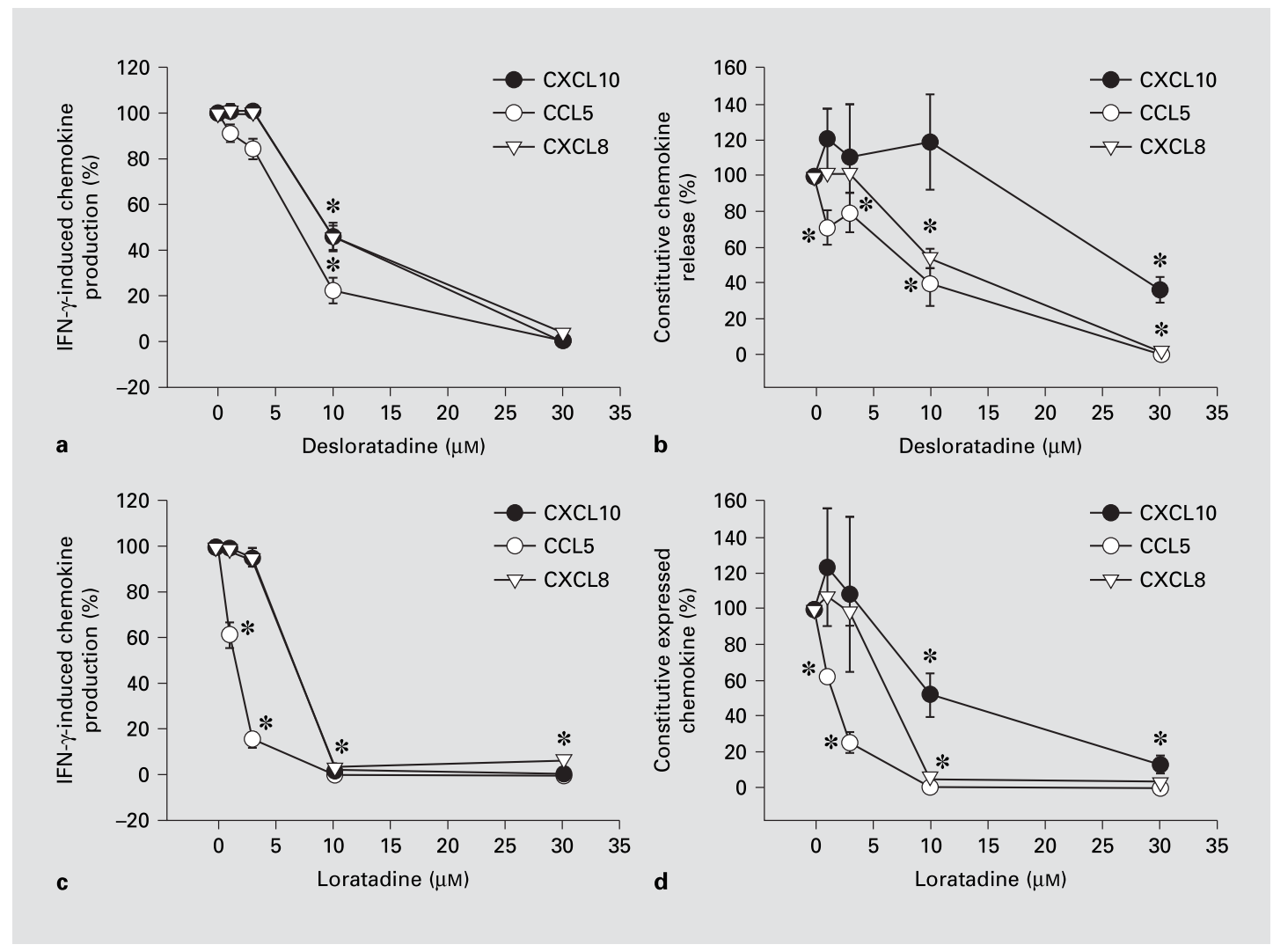

Fig. 1. Impaired chemokine release from primary keratinocytes in the presence of desloratadine $(\mathbf{a}, \mathbf{b})$ and loratadine $(\mathbf{c}, \mathbf{d})$. The keratinocytes were preincubated with desloratadine or loratadine for $4 \mathrm{~h}$ and then stimulated with IFN- $\gamma$ or medium. The supernatants were taken after $24 \mathrm{~h}$ of IFN- $\gamma$ stimulation and analyzed for the content of CCL5, CXCL10 and CXCL8. Incubation of keratinocytes with the antihistamines preceding the stimulation with IFN- $\gamma\left(300 \mathrm{U} \cdot \mathrm{ml}^{-1}\right)$ dose-dependently inhibited the release of the chemokines CCL5, CXCL8 and CXCL10 (a, c). The constitutive release of CCL5, CXCL10 and CXCL8 was similarly impaired (b, d). The results show the mean of pooled data \pm SEM from 7 independent experiments with keratinocytes from 5 different donors The data are presented as percentage of IFN- $\boldsymbol{\gamma}$-induced $\left(\mathrm{x}_{1}\right)$ or constitutional $\left(\mathrm{x}_{2}\right)$ chemokine production in order to equalize experiment-specific variabilities (CCL5 $\mathrm{x}_{1}=2,095.6 \pm 444 \mathrm{pg} / \mathrm{ml}, \mathrm{x}_{2}=$ $24.6 \pm 13 \mathrm{pg} / \mathrm{ml} ;$ CXCL8 $\mathrm{x}_{1}=167.9 \pm 25 \mathrm{pg} / \mathrm{ml}, \mathrm{x}_{2}=162 \pm$ $21.6 \mathrm{pg} / \mathrm{ml} ;$ CXCL10 $\mathrm{x}_{1}=997.4 \pm 145.91 \mathrm{pg} / \mathrm{ml}, \mathrm{x}_{2}=37.2 \pm$ $35.2 \mathrm{pg} / \mathrm{ml}$ ). Significant reductions of the IFN- $\gamma$-induced or constitutional chemokine production are indicated $\left({ }^{*} \mathrm{p} \leq 0.05\right)$ both loratadine and desloratadine, whereas CCL5 was dramatically blocked especially by loratadine $\left(\mathrm{IC}_{50}\right.$ $2 \mu \mathrm{M}$ for loratadine vs. $7 \mu \mathrm{M}$ for desloratadine). CCL17 was hardly detected in IFN- $\gamma$-stimulated keratinocytes $(3.5 \pm 0.2 \mathrm{pg} / \mathrm{ml})$, while the nonstimulated keratinocytes showed no detectable levels of CCL17 in culture supernatants. The very low IFN- $\gamma$-induced CCL17 release was not significantly changed, neither by desloratadine nor by loratadine. Annexin V/propidium staining confirmed that the toxic effects of desloratadine and loratadine in the concentrations investigated were not responsible for the reduction of chemokine production (data not shown).

\section{Keratinocytes Treated with Desloratadine or} Loratadine Show Impaired Capacity to Attract Neutrophils, Eosinophils and T Cells

In order to investigate whether the block of chemokine production (fig. 1) led to an impairment of the migration of inflammatory cells, supernatants of primary human keratinocyte cultures stimulated with IFN- $\gamma$ after prior incubation with loratadine and desloratadine were taken for migration studies after $48 \mathrm{~h}$. A direct effect of the antihistamines on the migration of granulocytes or lymphocytes was excluded in this experimental setup by a medium change and lavage after $24 \mathrm{~h}$, for all conditions including the IFN- $\gamma$ control. As documented in figure $2 \mathrm{a}$ and $\mathrm{b}$ des- 


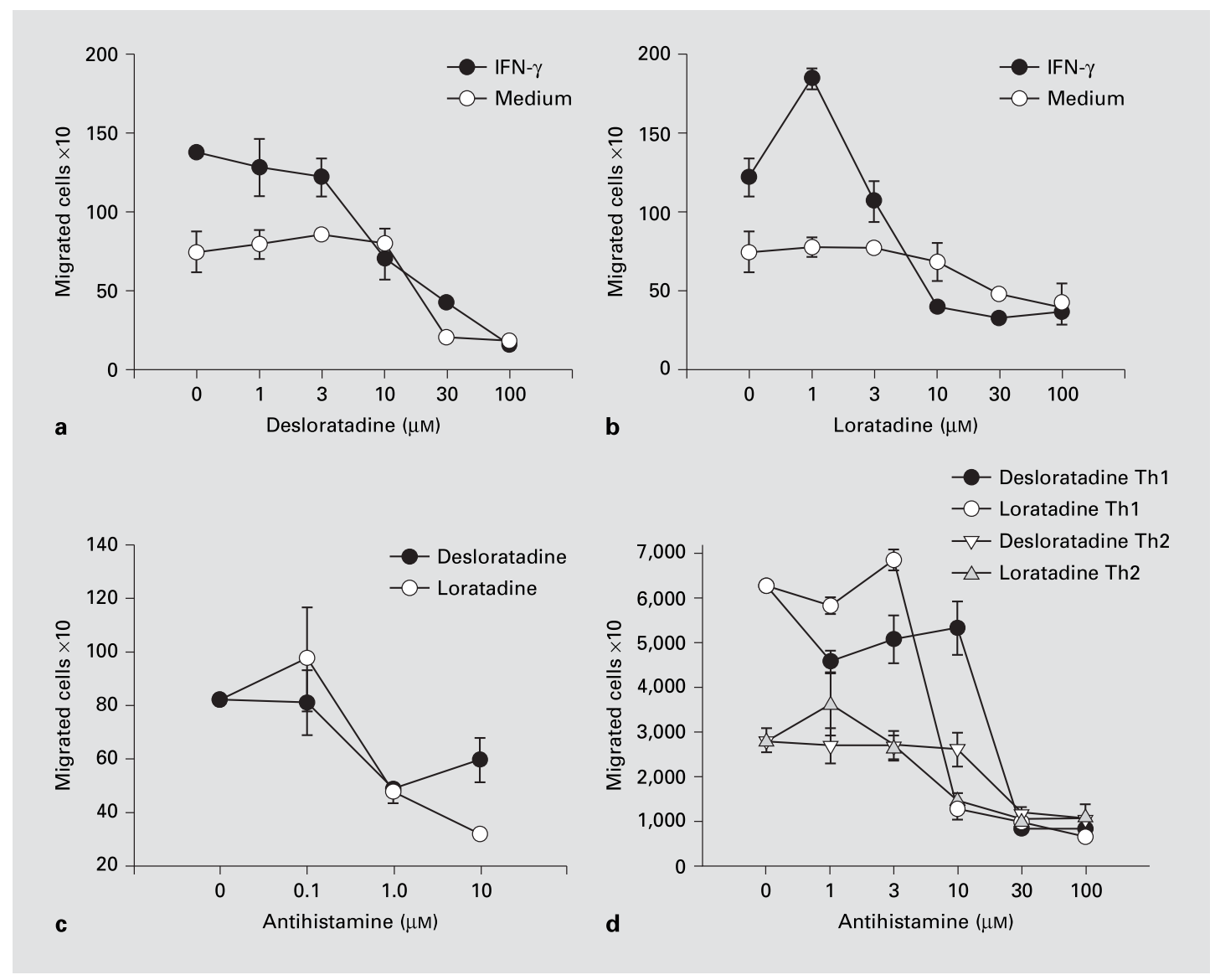

Fig. 2. Desloratadine and loratadine block the chemoattraction of neutrophils, eosionphils and T cells by human keratinocytes. The keratinocytes were stimulated with IFN- $\gamma$ for $24 \mathrm{~h}$ with or without prior incubation $(4 \mathrm{~h})$ with different concentrations of loratadine or desloratadine. The medium was changed after $24 \mathrm{~h}$ and left for another $24 \mathrm{~h}$ in order to exclude a direct effect of the antihistamines on the migrated cells. Then the supernatants were harvested, filtered sterile (to get rid of cells) and taken for migration studies. Desloratadine (a) and loratadine (b) dose-dependently blocked the release of constitutive (medium; significant reduction from $30 \mu \mathrm{M}$ antihistamine on) or IFN- $\gamma$-induced production (significant reduction from $10 \mu \mathrm{M}$ antihistamine on) of neutrophil chemoattractants. c Desloratadine and loratadine block the migration of human eosionphils induced by IFN- $\boldsymbol{\gamma}$-stimulated keratinocytes (significant reduction from $1 \mu \mathrm{M}$ antihistamine on). $\mathbf{d}$ Desloratadine and loratadine block the migration of Th1 and Th2 cells towards keratinocyte supernatants. The total migrated cells are shown. loratadine and loratadine both inhibited the release of constitutive and IFN- $\gamma$-induced chemoattractants for human neutrophils. As shown in figure 1 CXCL8 was significantly inhibited by desloratadine and loratadine, indicating that CXCL8 in keratinocyte supernatants was primarily responsible for the induction of neutrophil migration. Furthermore figure $2 \mathrm{c}$ shows that the reduction of the chemokine release from desloratadine- or loratadine-prestimulated keratinocytes also leads to a reduction of the migration of human eosinophils. Interestingly CCL5, and consecutively the migration of human eosinophils towards supernatants of loratadine or deslo- ratadine pretreated keratinocytes, was the most sensitive parameter (significant reduction of the migration already at $1 \mu \mathrm{M}$ of antihistamine) in this experimental setting, pointing to an especially interesting antiallergic activity of desloratadine and loratadine. In order to investigate the effect of the antihistamines on the keratinocyte-induced migration of T cells, polarized Th1 and Th2 cell clones were used for migration studies. First of all an attraction primarily of Th1 cells by IFN- $\gamma$-stimulated keratinocytes was observed (migration index of 10 for Th1 vs. 2.5 for Th2). Desloratadine and loratadine dose-dependently reduced the migration of Th1 and Th2 cells towards IFN- 


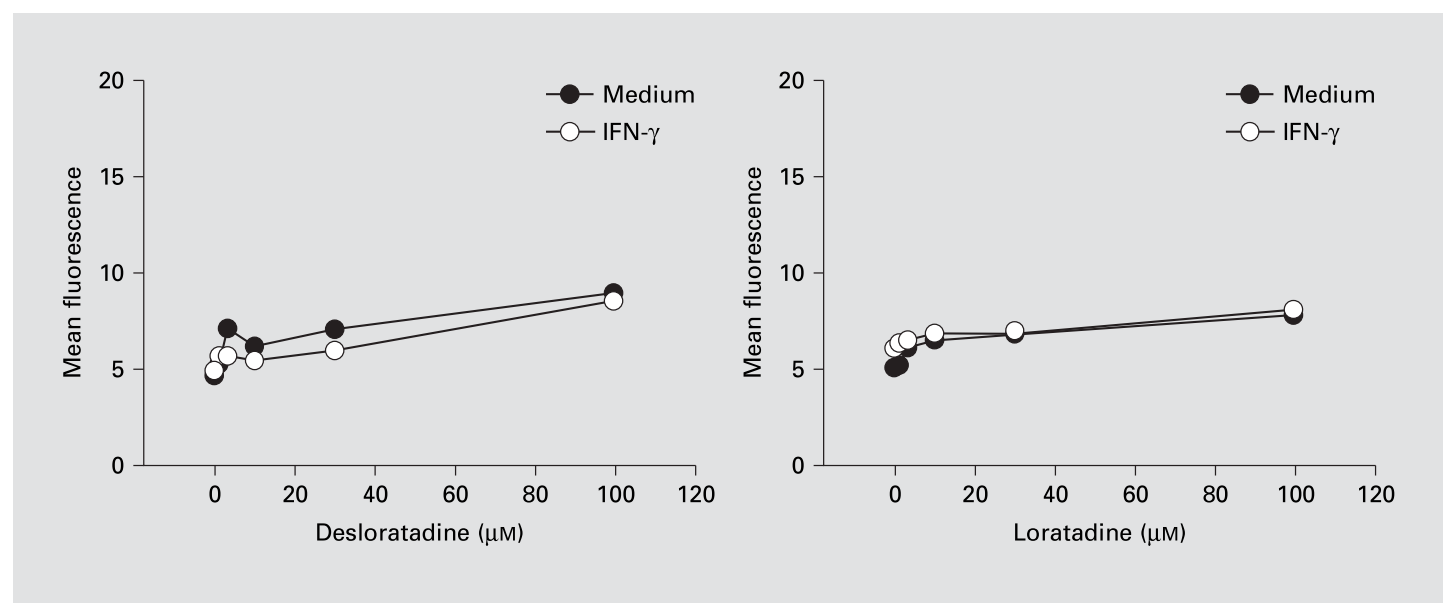

Fig. 3. Desloratadine and loratadine do not show toxicity in concentrations of the anti-inflammatory range. The keratinocytes were pretreated $4 \mathrm{~h}$ with desloratadine and further stimulated with IFN- $\gamma$ or medium for $24 \mathrm{~h}$ and subsequently stained with PI. No significant PI positivity was observed in the concentrations used in our experiments $(0.1-30 \mu \mathrm{M})$. One representative experiment out of 3 performed is shown.

$\gamma$-stimulated keratinocytes. Loratadine showed 90\% (and significant) inhibition of the Th1 migration at $10 \mu \mathrm{M}$, whereas desloratadine showed comparable effects at $30 \mu \mathrm{M}$. Nonstimulated keratinocytes did not lead to a significant migration of T cells, neither Th1 nor Th2 (data not shown).

\section{Exclusion of Toxic Effects of Desloratadine and \\ Loratadine}

In order to exclude toxic effects of desloratadine and loratadine leading to the effects described above, toxicity tests were performed. The keratinocytes were stimulated with desloratadine and loratadine ( $4 \mathrm{~h}$ preincubation with desloratadine/loratadine and further stimulation with IFN- $\gamma$ or medium alone) and tested for toxicity parameters (PI incorporation) after $24 \mathrm{~h}$. The keratinocytes were stained with PI and analyzed by flow cytometry. As shown in figure 3 there was only a marginal induction of necrosis $(100 \mu \mathrm{M})$. As documented in figures 1 and 2 the $\mathrm{IC}_{50} \mathrm{~s}$ for the observed anti-inflammatory effects ranged around 3-30 $\mu \mathrm{M}$, thus ruling out toxic effects of desloratadine and loratadine as an explanation of the observed phenomena.

\section{Discussion}

Herein we show that loratadine and its metabolite desloratadine significantly inhibit the constitutive and IFN$\gamma$-induced release of CXC and CC chemokines. This ob- servation was confirmed by functional studies showing that the chemotactic activity of IFN- $\gamma$-stimulated keratinocytes towards granulocytes and lymphocytes was reduced when prestimulated with desloratadine or loratadine. Comparing the potency desloratadine and loratadine seemed to have similar effects. This observation does not go in line with the recent description of the higher affinity of desloratadine to the histamine receptor compared to loratadine. On the other hand it suggests that the inhibition of the chemokine release by these antihistamines is not exclusively linked to an action on the histamine receptor. Recently the effect of histamine on keratinocytes was investigated [13]. Here an effect of levocetirizine on the IFN- $\gamma$-induced but not on the histamine-induced CCL5/RANTES and granulocytemacrophage colony-stimulating factor release was observed. A similar activity has been demonstrated for cetirizine [14] and may be the consequence of the inhibition of endogenous histamine produced by keratinocytes in response to IFN- $\gamma$.

Wu et al. [15] recently demonstrated that desloratadine had an inhibitory effect on the constitutive and histamine-induced NF- $\kappa$ B activation. Since NF- $\mathrm{KB}$ is responsible for many immunoregulatory genes including CXCL8 and CCL5 [16], the inhibitory effects of loratadine and desloratadine on NF- $\mathrm{\kappa B}$ signaling could play a role in the constitutive release of CXCL8 and CCL5. However, IFN- $\gamma$ uses different signaling pathways, based primarily on the activation cascade of Janus kinases and STAT (signal 
transducers and activators of transcription) molecules. Consequently the inhibition of the IFN- $\gamma$ induced production of CXCL10, CXCL8 and CCL5 is most likely partly due to another pathway independent of NF- $\mathrm{KB}$.

In conclusion the results of our study suggest that desloratadine can inhibit the IFN- $\gamma$-induced activation of cultured keratinocytes, which represents an in vitro model of Th1-mediated skin inflammation. Even though the concentrations of the drug required for inhibiting CCL5, CXCL10 and CXCL8 are higher than those thought to be achieved in the plasma, following a regular oral intake of $10 \mathrm{mg} /$ day $[17,18]$, the immunomodulatory effects of lo- ratadine and desloratadine are striking. These observations propose to revalue the development of topical administration of antihistamine in the acute or chronic phase of inflammatory disorders of the skin.

\section{Acknowledgments}

The excellent technical assistance of Simone Knabl and Gabi Pleyl-Wisgickl is gratefully acknowledged. C.T.-H. was a recipient of the Bayerische Habilitationsförderpreis.

\section{References}

1 Haria M, Fitton A, Peters DH: Loratadine: a reappraisal of its pharmacological properties and therapeutic use in allergic disorders. Drugs 1994;48:617-637.

2 Ring J, Hein R, Gauger A: Desloratadine in the treatment of chronic idiopathic urticaria. Allergy 2001;56(suppl 65):28-32.

3 Kleine-Tebbe J, Josties C, Frank G, Stalleicken D, Buschauer A, Schunack W, Kunkel G, Czarnetzki B: Inhibition of IgE- and non-IgE-mediated histamine release from human basophil leukocytes in vitro by a histamine H1-antagonist, desethoxycarbonyl-loratadine. J Allergy Clin Immunol 1994;93:494500.

4 Assanasen P, Naclerio RM: Antiallergic antiinflammatory effects of $\mathrm{H1}$-antihistamines in humans. Clin Allergy Immunol 2002;17:101139.

5 Lugovic L, Lipozenic J, Jakic-Razumovic J: Prominent involvement of activated Th1 sub set of $\mathrm{T}$ cells and increased expression of receptor for IFN- $\gamma$ on keratinocytes in atopic dermatitis acute skin lesions. Int Arch Allergy Immunol 2005;137:125-133.

6 Albanesi C, Cavani A, Girolomini G: Interferon-stimulated human keratinocytes express the genes necessary for the production of peptide-loaded MHC class II molecules. J Invest Dermatol 1998;75:138-142.
7 Pastore S, Mascia F, Maritati F, Dattilo C, Girolomoni G: Chemokine networks in inflammatory skin diseases. Eur J Dermatol 2004;14: 203-208.

8 Albanesi C, Scarponi C, Sebastiani S, Cavani A, Federici M, Sozzoni S, Girolomini G: A cytokine-to-chemokine axis between T lymphocytes and keratinocytes can favor Th1 cell accumulation in chronic inflammatory skin diseases. J Leukoc Biol 2001;70:617-623.

9 Lippert U, Kruger-Krasagakes S, Moller A, Kiessling U, Czarnetzki BM: Pharmacological modulation of IL- 6 and IL- 8 secretion by the $\mathrm{H} 1$-antagonist decarboethoxy-loratadine and dexamethasone by human mast and basophilic cell lines. Exp Dermatol 1995;4:272-276.

10 Traidl C, Sebastiani S, Albanesi C, et al: Disparate cytotoxic activity of nickel-specific CD8+ and CD4+ T cell subsets against keratinocytes. J Immunol 2000;165:3058-3064.

11 Traidl-Hoffmann C, Kasche A, Jakob T, Huger M, Plotz S, Feussner I, Ring J, Behrendt $\mathrm{H}$ : Lipid mediators from pollen act as chemoattractants and activators of polymorphonuclear granulocytes. J Allergy Clin Immunol 2002; 109:831-838.

12 Plötz SG, Traidl-Hoffmann C, Feussner I, Kasche A, Feser A, Ring J, Jakob T, Behrendt $\mathrm{H}$ : Chemotaxis and activation of human peripheral blood eosinophils induced by pollenassociated lipid mediators. J Allergy Clin Immunol 2004;113:1152-1160.
13 Giustizieri ML, Albanesi C, Fluhr J, Gisondi P, Norgauer J, Girolomoni G: H1-histamine receptor mediates inflammatory responses in human keratinocytes. J Allergy Clin Immunol 2004;114:1176-1182.

14 Albanesi C, Pastore S, Fanales-Belasio E, Girolomoni G: Cetirizine and hydrocortisone differentially regulate ICAM-1 expression and chemokine release in cultured human keratinocytes. Clin Exp Allergy 1998;28:101-109.

$15 \mathrm{Wu}$ RL, Anthes JC, Kreutner W, Harris AG, West RE Jr: Desloratadine inhibits constitutive and histamine-stimulated nuclear factor$\kappa \mathrm{B}$ activity consistent with inverse agonism at the histamine H1-receptor. Int Arch Allergy Immunol 2004;135:313-318.

16 Baldwin SA: The NF-B and IBB proteins: new discoveries and insights. Annu Rev Immunol 1996;14:649-681

17 Michel L, De Vos C, Rihoux JP, Burtin C, Benveniste J, Dubertret L: Inhibitory effect of oral desloratadine on in vivo antigen-induced histamine and PAF-acether release and eosinophil recruitment in human skin. J Allergy Clin Immunol 1988;82:101-109.

18 Simons FE, Murray HE, Simons KJ: Quantitation of H1-receptor antagonists in skin and serum. J Allergy Clin Immunol 1995;95:759764 